\title{
Tribological and Mechanical Characterization of Carbon-Coated Sliders and Disks
}

\author{
R. Brunner • F. E. Talke
}

Received: 27 July 2009/ Accepted: 28 September 2009/Published online: 15 October 2009

(C) The Author(s) 2009. This article is published with open access at Springerlink.com

\begin{abstract}
Tribological and mechanical properties of carbon-coated sliders and disks manufactured by various vendors were evaluated using atomic force microscopy, Raman spectroscopy, X-ray photoelectron spectroscopy, and nano-indentation testing in the presence of ultrasonic excitation. The Raman spectra of carbon-coated sliders were found to differ significantly from the Raman spectra of carbon-coated disks. The tribological properties of carboncoated commercially available sliders did not show substantial differences regardless of the manufacturer. The same result was observed for commercially available disks manufactured by different manufacturers. Dynamic nanoindentations in the presence of ultrasonic excitation indicate discontinuities which are correlated to overcoat thickness.
\end{abstract}

Keywords Carbon overcoat - Raman spectroscopy · Atomic force microscopy $\cdot$ Roughness measurement

\section{Introduction}

Magnetic recording heads and disks are typically protected by an ultra-thin carbon film on the order of $3 \mathrm{~nm}$ or less in thickness. To achieve a storage density of $1 \mathrm{Tbit} / \mathrm{inch}^{2}$, a reduction in the magnetic spacing (distance between the magnetic layer on the disk and the pole piece of the readwrite head) between slider and disk is required [1]. Thus, assuming a nominal flying height of $3 \mathrm{~nm}$, and a lubricant thickness of $1 \mathrm{~nm}$, the thickness of the carbon overcoat must be on the order of $1 \mathrm{~nm}$ or less. In addition, the

R. Brunner $(\bowtie) \cdot$ F. E. Talke

CMRR, University of California, San Diego, 9500 Gilman

Drive, La Jolla, CA 92093, USA

e-mail: rbrunner@talkelab.ucsd.edu; rbrunner@ucsd.edu overall roughness of the disk needs to be reduced to allow an increase in storage density.

The mechanical properties of thin carbon films can be studied using nano-indentation and nano-scratch testing. Tribological performance of carbon films has also been investigated using scanning probe microscopy (SPM) [2]. In addition, ultrasonically excited nano-indentation testing can be used to provide an accelerated wear and fatigue test at a local contact [3].

Raman spectroscopy can be used to probe the structure of carbon films and reveal information about morphology [4]. Raman spectroscopy can also be used to measure the thickness of carbon films in the range from 10 to $50 \mathrm{~nm}[5,6]$.

In order to obtain satisfactory mechanical performance of the head-disk interface with films on the thickness of $1 \mathrm{~nm}$, it is imperative to establish a fundamental understanding between carbon film structure and performance. The properties of the interface between the carbon film and the lubricant film are also of importance for the tribological performance of the head/disk interface.

This paper investigates the tribological properties of ultrathin carbon overcoats currently used in sliders and disks. In particular, nano-scratch testing was used to characterize the mechanical properties and wear characteristics of the films. We also performed dynamic nano-indentation studies in the presence of ultrasonic excitation to investigate dynamic properties of presently used carbon overcoats for sliders and disks. Finally, Raman spectroscopy was used to identify the ratio of $\mathrm{sp}^{2}$ - and $\mathrm{sp}^{3}$-bonds in the carbon films [7].

\section{Disk and Sliders Materials}

The disks studied were commercially available 3.5 inch $(95 \mathrm{~mm})$ hard disks from different vendors. Typically, the 
magnetic layer is deposited by either sputtering or chemical vapor deposition onto a nickel phosphorus-plated aluminum/magnesium substrate. The sliders used in this investigation are made by standard etch procedures using semiconductor manufacturing processes. The material of all sliders is $\mathrm{Al}_{2} \mathrm{O}_{3}-\mathrm{TiC}$ (alumina titanium carbide).

Both, the disks and sliders are coated with a protective carbon overcoat. In addition, the disks were dip-coated with a thin lubricant layer. Sputtering, chemical vapor deposition and arc-discharge techniques are typical deposition methods for the wear protective carbon overcoat. The structure of the deposited carbon film is strongly dependent on the method and conditions employed during film deposition. The amorphitization stage in the carbon overcoat is changing from amorphous carbon (a-C) to tetrahedral amorphous carbon (ta-C) depending on the process conditions used [4]. As a consequence, the mechanical and tribological properties of the films can change with changes in the $\mathrm{sp}^{2}$ and $\mathrm{sp}^{3}$ content.

\section{AFM Roughness Measurements on Disk and Slider Surfaces}

Atomic force microscopy (Veeco-Dimension 3000) in close contact mode (dynamic mode) was used to determine the roughness of a number of commercially available disks and sliders. The root-mean-square (Rms) roughness was determined on an area of $10 \times 10 \mu \mathrm{m}^{2}$. A typical 3-dimensional image of the surface topography of a carbon-coated disk is shown in Fig. 1.

The hard disks used in this experiment were obtained from various vendors and were manufactured between 2004 and 2008. Figure 2 shows the Rms roughness for the various disks, indicating a decrease in Rms roughness from $0.55 \mathrm{~nm}$ to $0.15 \mathrm{~nm}$, in the time period from 2004 to 2008 . Disk \#1 has the highest Rms roughness and was manufactured in 2004, while disk \#7 with the lowest Rms

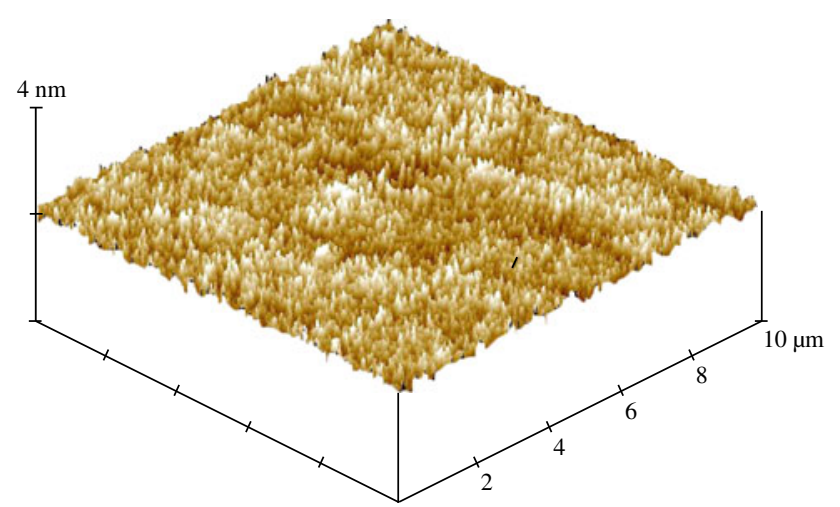

Fig. 1 3D topography image of a smooth magnetic hard disk

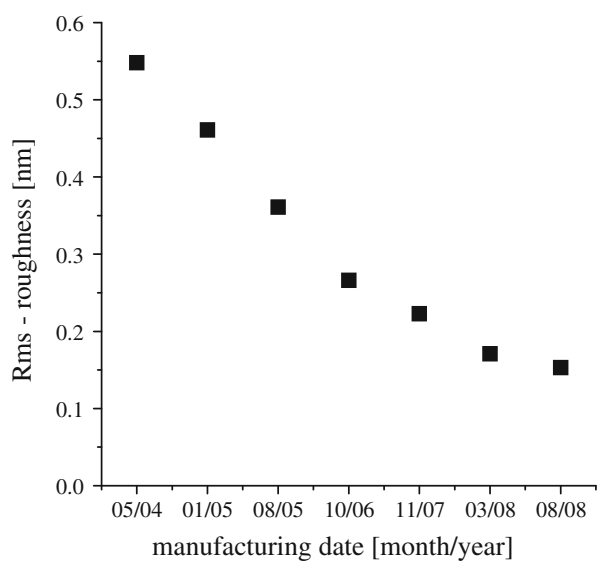

Fig. 2 Comparison of RMS roughness of magnetic hard disk surfaces

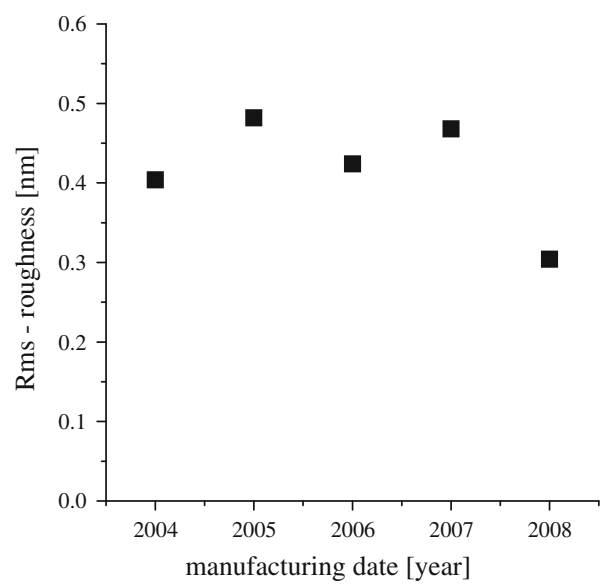

Fig. 3 Comparison of RMS roughness of slider surfaces

roughness, was manufactured in 2008. Clearly, disks have become so smooth that the Rms roughness is on the order of the atomic distance between two carbon atoms.

The Rms roughness for the sliders used in this study was found to be between 0.3 and $0.5 \mathrm{~nm}$ (see Fig. 3).

\section{Nano-Indentation and Nano-Scratch Testing}

The qualitative characterization of the mechanical properties of the surface of commercially available sliders and disks was performed using a 2D-lateral force transducer (Triboscope ${ }^{\circledR}$, Hysitron, Inc.) in conjunction with a SPM stage (NanoScope ${ }^{\circledR}$, Digital Instruments). Nano-indentation and nano-scratch testing was used to determine the relative hardness and the friction coefficient, respectively. For both sets of measurements, a cube corner diamond tip was used and the normal force applied to the surface was varied between 30 and $70 \mu \mathrm{N}$. A triangular force profile with $20 \mathrm{~s}$ loading cycle to the maximum applied force and subsequent $20 \mathrm{~s}$ unloading of the tip was applied. 
Figure 4 shows a 3D SPM image of the nano-indentation on a hard disk. The contact depth on the disks and sliders exceeded the thickness of the carbon films, i.e., the hardness measurements correspond to hardness values of the underlying substrate rather than the hardness of the thin carbon film. Figure $5 \mathrm{a}$ and $\mathrm{b}$ shows the hardness measured for a number of disks and sliders, respectively. We observe that the hardness values are between 7 and $8 \mathrm{GPa}$ for the disks and approximately $10 \mathrm{GPa}$ for the various sliders. For the same normal force of $70 \mu \mathrm{N}$, the depth of the nanoindentation was approximately $3 \mathrm{~nm}$ for the sliders and approximately $8 \mathrm{~nm}$ for the disks. The higher hardness in the case of the sliders is related to the harder slider substrate material $\left(\mathrm{Al}_{3} \mathrm{O}_{2}-\mathrm{TiC}\right)$.

Nano-scratch tests were also performed on conventional hard disks and sliders. The normal force, lateral force, and resulting friction coefficients were measured. During the

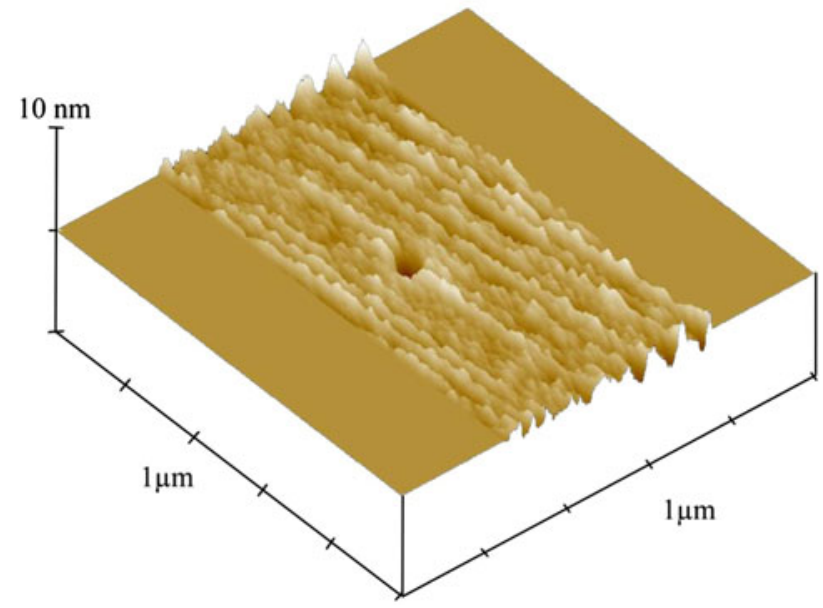

Fig. 4 3D SPM image of a nano-indentation on a hard disk

Fig. 5 Hardness measurements on typical a hard disks and $\mathbf{b}$ sliders using nano-indentation scratch testing the normal force applied to the cube corner diamond tip was increased from 0 to $70 \mu \mathrm{N}$. During the scratch test the tip traveled approximately $4 \mu \mathrm{m}$ in $40 \mathrm{~s}$. Figure 6 shows a SPM image of a typical nano-scratch test on a hard disk. Penetration into the surface occurs at loads above $35 \mu \mathrm{N}$, equivalent to a distance of $2 \mu \mathrm{m}$ along the scratch. Figure $7 \mathrm{a}$ and $\mathrm{b}$ shows the friction coefficient from the nano-scratch measurements at 35,50 , and $70 \mu \mathrm{N}$ for the different disks and sliders, respectively. The friction coefficient was found to be a slight function of the load. For a load of $35 \mu \mathrm{N}$, the friction coefficient was approximately 0.17 for the disks investigated, while at a load of $70 \mu \mathrm{N}$ the friction coefficient increased to a value between 0.2 and 0.23 . As the load increases, the indentation depth during scratching increases (see Fig. 6).

The friction coefficient for the sliders investigated (Fig. 7b) was found to be between 0.25 and 0.4 . It increased slightly at loads higher than $35 \mu \mathrm{N}$. The increase in the friction coefficient seems to be related to plastic

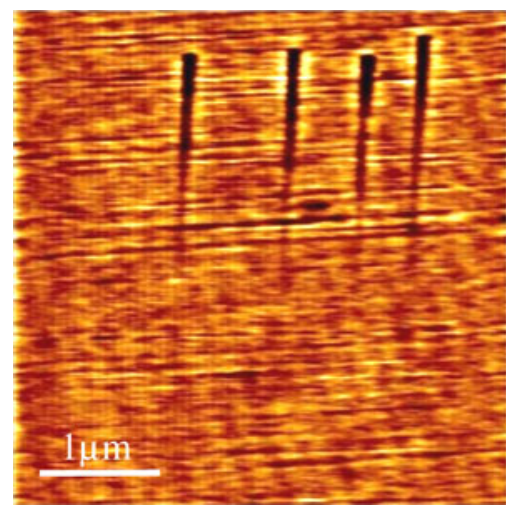

Fig. 6 SPM image of nano-scratches on a carbon-coated hard disk

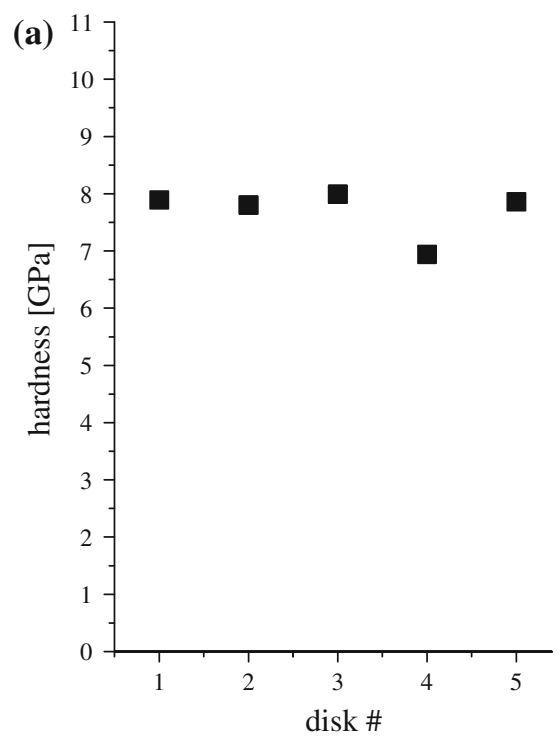

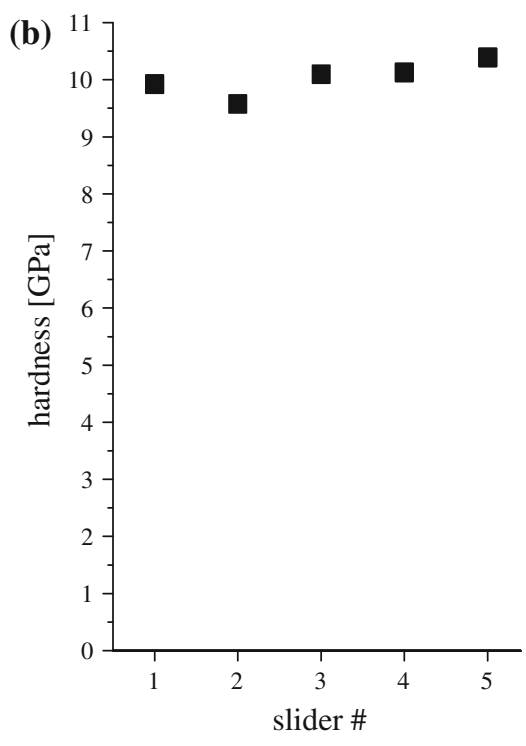



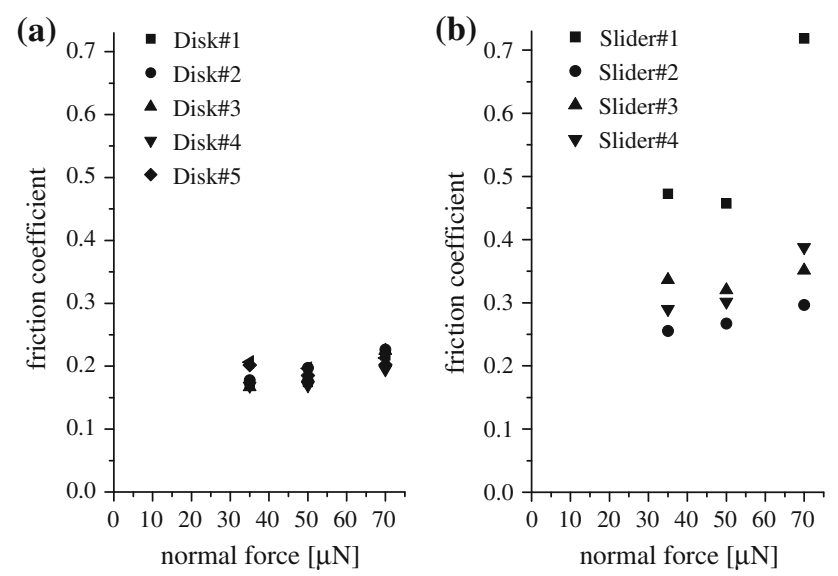

Fig. 7 Friction coefficient measurements of carbon-coated disks (a) and slides (b) at 35, 50, and $70 \mu \mathrm{N}$ using nano-scratch tests

deformation during scratching. Comparing the films on the sliders with the films on the disks, we observe noticeable differences. On the other hand, comparing the films on all sliders with each other, we conclude that their properties are very similar. The same can be said when comparing the films on the various disks. Thus, it seems justifiable to postulate that the films on the disks were manufactured using a similar process. In addition, the films on the sliders seem to have been manufactured by the same process in spite of the fact that they were manufactured by different vendors.

Fig. 8 Nano-indentation a without ultrasonic excitation and $\mathbf{b}$ in the presence of ultrasonic excitation, at $35 \mu \mathrm{N}$ maximum force

Fig. 9 SPM images of nanoindentation at $35 \mu \mathrm{N}$. (a) without ultrasonic excitation, (b) with ultrasonic excitation

\section{Nano-Indentation in the Presence of Ultrasonic Excitation}

Accelerated wear testing of carbon-coated sliders was performed using nano-indentation in the presence of ultrasonic excitation. An ultrasonic piezoelectric transducer (PZT) was used to generate a sinusoidal displacement in the direction of the indentation normal to the surface. The slider was securely glued onto the PZT to perform the tests. A driving frequency between 101 and $103 \mathrm{kHz}$ was used with displacement amplitude of 1 to $2 \mathrm{~nm}$. The specific setup is described in more detail in [3]. The indentation force was held constant at $30 \mu \mathrm{N}$ for all measurements.

Figure 8a shows the force-displacement curve for an indentation measurement on slider \#2 without ultrasonic excitation. The maximum indentation depth was found to be approximately $4.5 \mathrm{~nm}$ and the in situ SPM image in Fig. 8a did not show any physical evidence of an indentation.

Figure $8 \mathrm{~b}$ shows a force-distance curve of a nanoindentation performed in the presence of ultrasonic excitation. The indentation depth is significantly larger than for the static measurement at the same normal load of $30 \mu \mathrm{N}$. A clear indentation mark is visible in the SPM image in Fig. 9. Discontinuities in the force displacement curve are observed during the loading cycle (see Fig. 8). It is likely that these discontinuities are related to fatigue of the interface between the carbon film and the $\mathrm{Al}_{3} \mathrm{O}_{2}-\mathrm{TiC}$
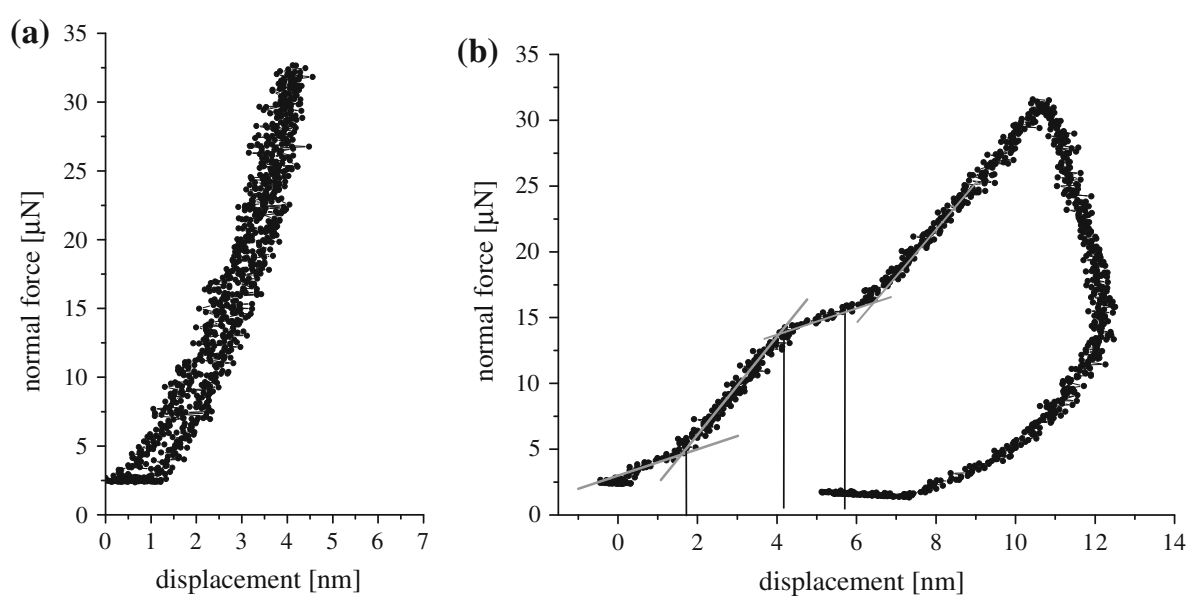
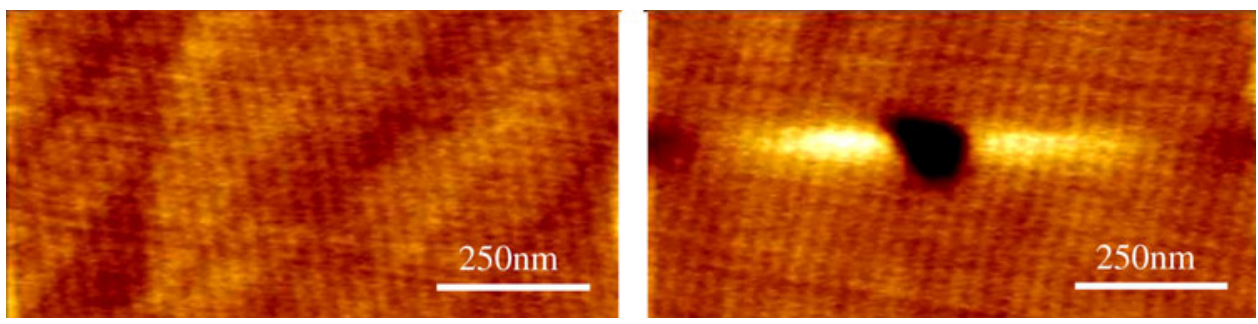
Table 1 Indentation depth of first and second discontinuity for the different sliders

\begin{tabular}{lllll}
\hline Slider & $\# 2(\mathrm{~nm})$ & $\# 3(\mathrm{~nm})$ & $\# 4(\mathrm{~nm})$ & $\# 5(\mathrm{~nm})$ \\
\hline 1st Discontinuity & 2 & 4 & 3 & 2 \\
2nd Discontinuity & 7 & 8 & 7 & 5 \\
\hline
\end{tabular}

substrate. During cyclic loading, delamination from the substrate can occur due to fatigue in the substrate-carbon overcoat interface. This results in discontinuities in the force displacement curve [3]. Figure $9 \mathrm{~b}$ shows delamination or pile up to the left and right (bright half-oval area) of the indentation. The first discontinuity occurs at an indentation depth of approximately $2 \mathrm{~nm}$ or $3 \mathrm{~nm}$, corresponding to the thickness of the carbon overcoat. We hypothesize that this is the indentation depth when the tip initially breaks through the carbon layer. The second discontinuity at an indentation depth of about $7 \mathrm{~nm}$ is assumed to correspond to conditions where the carbon overcoat starts to delaminate.

The results of the indentation depths obtained for the first and second discontinuities are summarized in Table 1.

\section{Raman Spectroscopy}

Raman spectroscopy is a technique to study vibrational, rotational, and other low-frequency modes of atoms and molecules in material systems. The spectra can give information about bonding states in the carbon overcoat. In this study, a Raman spectrometer (Renishaw Raman microspectrometer) was used to investigate the structural parameters of different carbon overcoats of commercially available hard disks and sliders. The Raman spectrometer uses an argon excitation laser (Melles Griot) with a monochromatic laser light at $514.5 \mathrm{~nm}$ wavelength. The laser light is focused with the aid of an optical microscope on the specimen. The reflected light is sent through a spectral grid to a charged coupled device (CCD) line for detection of the spectra. A schematic of the Raman microspectrometer is shown in Fig. 10.

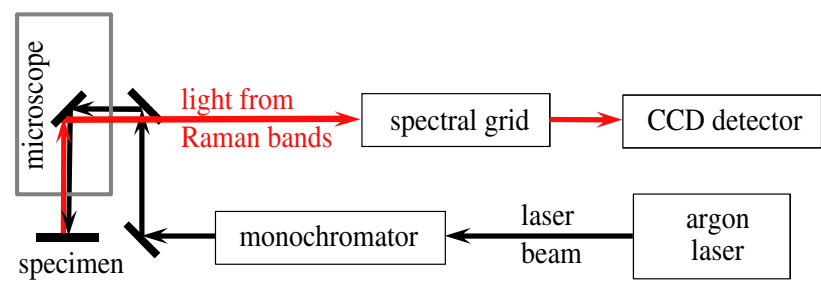

Fig. 10 Schematic of the Raman spectrometer

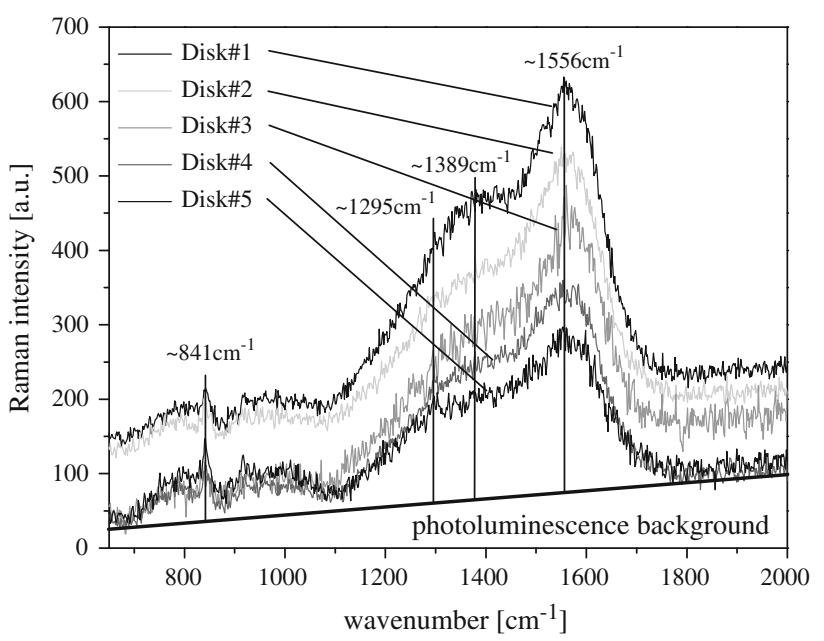

Fig. 11 Raman spectra of different commercially available disks

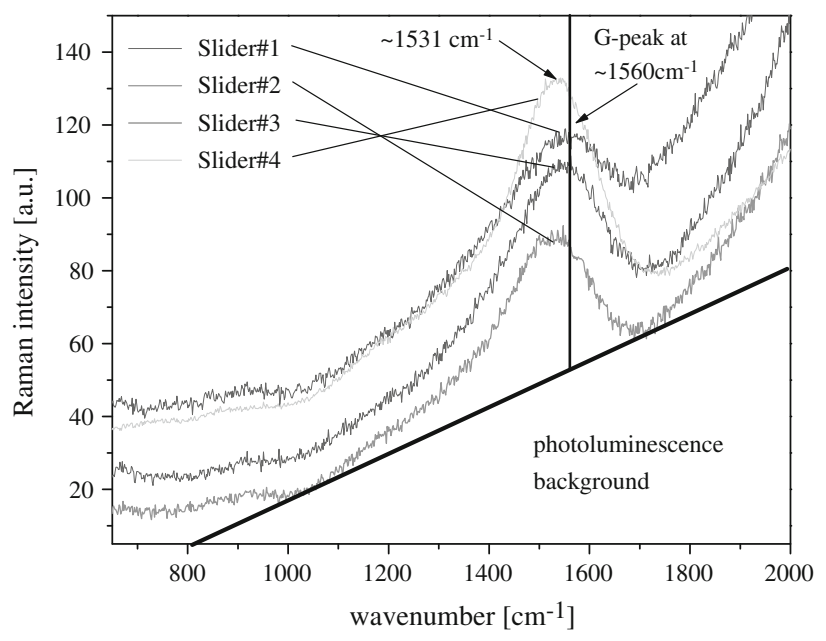

Fig. 12 Raman spectra of different commercially available sliders

Figures 11 and 12 show Raman spectra for the disks and sliders available in this study. From Fig. 11 we observe that the Raman spectra for the disks evaluated are similar. Each spectra show well-defined G-band and D-band peaks at wave numbers of 1560 and $1389 \mathrm{~cm}^{-1}$, respectively. The $I(\mathrm{D}) / I(\mathrm{G})$ ratio is between 0.55 and 0.65 . A comparison of the spectra with data by Robertson [4] suggests that the carbon is comprised of a high fraction of amorphous carbon (a-C) for the measured spectral profiles. The variation in the overall spectral intensity of the curves for the disks is caused by both laser beam misalignment and variation in the thickness of the carbon overcoats [5], [6], [8]. In addition, the photoluminescence background suggests that the nitrogen or hydrogen content is on the same order for all disks measured [9].

The Raman spectra of the sliders available for this study are shown in Fig. 12. We observe that the G-band is well 
pronounced for each spectrum but that the D-band peak is absent. This behavior is typical for a film with a tetrahedral amorphous structure with a high amount of $\mathrm{sp}^{3}$-bonding. The $\mathrm{sp}^{3}$-bonding increases at wave numbers above $1520 \mathrm{~cm}^{-1}$ [7]. The position of the G-peak for the different sliders varied between 1522 and $1560 \mathrm{~cm}^{-1}$. An increase in $\mathrm{sp}^{3}$-bonding, for example, due to an increase in hydrogen or nitrogen content in the carbon overcoat of the disks or the sliders can also add to the shift of the G-peak. This result is also supported by the increased slope of the photoluminescence background if one compares the spectra of the sliders (Fig. 12) with the spectra of the disks (Fig. 11) [9].

The Raman spectra in Figs. 11 and 12 show differences in the amorphitization stage between the amorphous carbon films on the disks and the tetrahedral amorphous carbon films on the sliders [4]. However, the difference between individual disks is small and it is apparent that all disks are manufactured in a similar way in spite that they were manufactured by different vendors. The same conclusion applies for the different sliders investigated.

\section{X-Ray Photoelectron Spectroscopy of Hard Disks}

X-ray photoelectron spectroscopy (XPS) was used to determine differences in the material compositions of different hard disks. X-rays were produced by $\mathrm{K} \alpha$ electron transitions of magnesium with an energy of $1253.6 \mathrm{eV}$. The $\mathrm{X}$-rays were focused on the sample in an analytic ultra-high vacuum chamber. The emitted electrons from the sample were captured in a half sphere analyzer to determine their kinetic energy.

Figure 13 shows overview XPS spectra of the same carbon-coated and lubricated magnetic disks investigated previously. We have shifted the spectra against each other for better clarity. Individual peaks as well as Auger

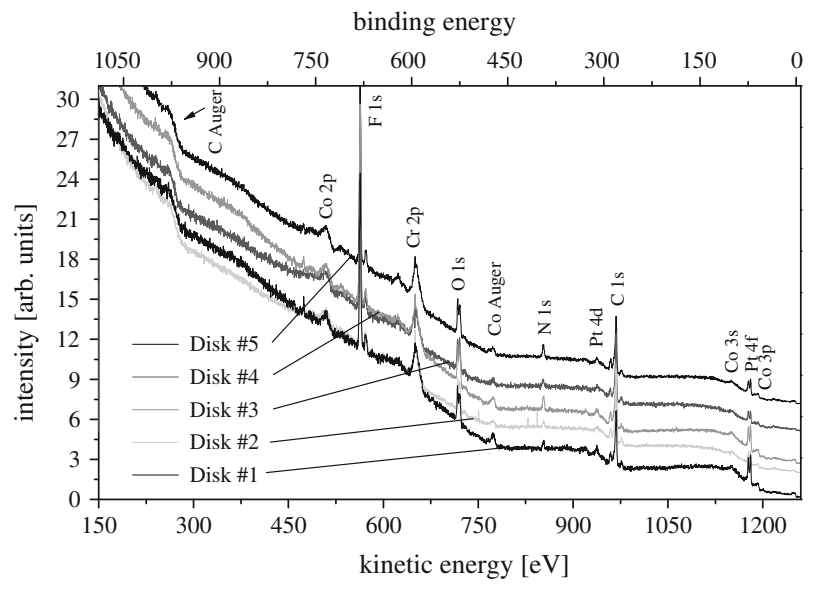

Fig. 13 XPS survey spectrum of a carbon-coated magnetic disk

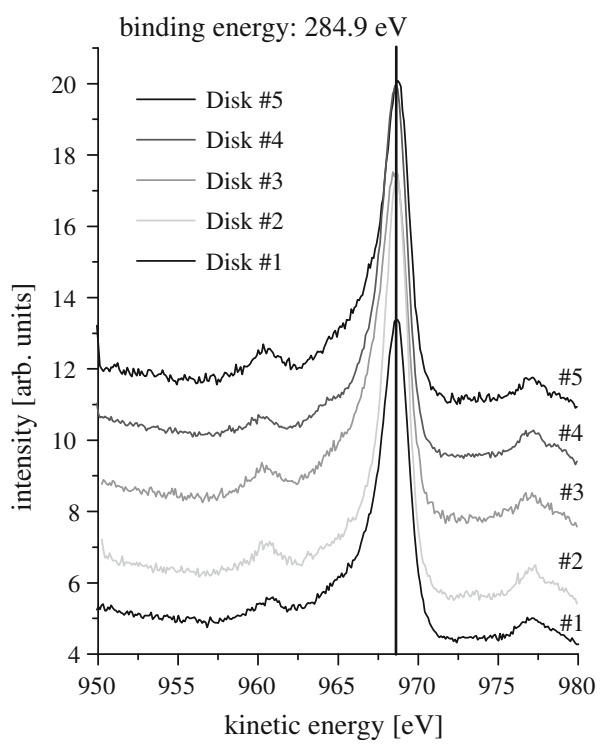

Fig. 14 High-resolution scan of the $\mathrm{C}$ 1s peak for the different disks

transitions of the magnetic layer ( $\mathrm{Co} 2 \mathrm{p}, \mathrm{Pt} 4 \mathrm{f}, \mathrm{Cr} 2 \mathrm{p}$ ), the carbon overcoat (C 1s) and the lubricant (F 1s, C 1s, O 1s) are visible. From Fig. 13 one can see that the composition of all disks is very similar as observed earlier. In addition, the $\mathrm{N}$ 1s peak indicates that the overcoat on all disks is a nitrogenated carbon film. From the spectrum overview we also observe that the magnetic layer for the disk sample consists of a Co-Pt-Cr alloy.

Figure 14 shows the high-resolution $\mathrm{C}$ 1s spectrum of carbon on the carbon-coated and lubricated magnetic disks. Using the high-resolution spectra of the individual peaks (in our case the $\mathrm{C} 1 \mathrm{~s}$ peak), we can detect a small peak shift. This shift gives information about the bonding state of the atoms which can be used to gather information about the carbon composition, film thickness, and even disk corrosion. From the disks studied, we observe that any differences, if they exist, are very small, i.e., it is likely that the disks were all manufactured in a similar way.

\section{Discussion and Summary}

Nano-indentation and nano-scratch measurements were performed to characterize the tribological properties of carbon-coated sliders and disks manufactured by various vendors. It was found that the materials and tribological properties of the various disks were comparable. The same result was observed for the properties of the carbon overcoats on various sliders. The hardness of the carbon-coated sliders was higher compared to the hardness of the carboncoated disks. The latter is a consequence of the fact that a harder substrate is used for sliders than for disks. The increase in the friction coefficient on disk and slider 
samples seems to be related to plastic deformation, fatigue and debris formation during the scratch tests.

Nano-indentations measurements in the presence of ultrasonic excitation indicate discontinuities during the load-cycle. This phenomenon appears at a displacement depth on the order of the carbon overcoat thickness and can possibly be used as a film thickness measurement technique.

Raman spectroscopy showed that only slight differences exist in the characteristics of the amorphous carbon overcoats for the disks investigated. The same result was also found using X-ray photoelectron spectroscopy. Somewhat larger differences were observed in the characteristics of the carbon films of the various sliders investigated, presumably related to slight differences in the tetrahedral amorphous carbon structure in the overcoat of the various sliders.

The results suggest that the manufacturing processes used by different vendors for sliders and disks are very similar.

Open Access This article is distributed under the terms of the Creative Commons Attribution Noncommercial License which permits any noncommercial use, distribution, and reproduction in any medium, provided the original author(s) and source are credited.

\section{References}

1. Li, D.J., Chung, Y.W.: Ultrasmooth $\mathrm{CNx}$ overcoats for nextgeneration hard disks. IEEE Trans. Magn. 39(2), 765-768 (2003)

2. Jacoby, B., Wienss, A., Ohr, R., von Gradowski, M., Hilgers, H.: Nanotribological properties of ultra-thin carbon coatings for magnetic storage devices. Surf. Coat. Technol. 174, 1126-1130 (2003)

3. Matsuda, Y., Yoon, Y., Brunner, R., Daugela, A., Warren, O.L., Talke, F.E.: Nano-hardness testing with ultrasonic excitation. Wear 259, 1497-1501 (2005)

4. Robertson, J.: Diamond-like amorphous carbon. Mater. Sci. Eng. R 37, 129-281 (2002)

5. Varanasi, S.S., Lauer, J.L., Talke, F.E., Wang, G., Judy, J.H.: Friction and wear studies of carbon overcoated thin films magnetic sliders: application of Raman microspectroscopy. J. Tribol. 119, 471 (1997)

6. Scharf, T.W., Singer, I.L.: Thickness of diamond-like carbon coatings quantified with Raman spectroscopy. Thin Solid Films 440, 138-144 (2003)

7. Ferrari, A.C.: Diamond-like carbon for magnetic storage disks. Surf. Coat. Technol. 180, 190-206 (2004)

8. Prabhakaran, V., Kohira, H., Talke, F.E.: Measurement of wear of carbon coated sliders using atomic force microscopy and Raman spectroscopy. Trib. Intern. 33, 655-663 (2000)

9. Neuhaeuser, M., Hilgers, H., Joeris, P., White, R., Windeln, J.: Raman spectroscopy measurements of DC-magnetron sputtered carbon nitride $(\mathrm{a}-\mathrm{C}: \mathrm{N})$ thin films for magnetic hard disk coatings. Diam. Relat. Mater. 9, 1500-1505 (2000) 